\title{
Conformal bootstrap and the $\lambda$-point specific heat experimental anomaly
}

\author{
Carving out OPE space and precise $\mathrm{O}(2)$ model critical exponents \\ Authors: S. M. Chester, W. Landry, J. Liu, D. Poland, D. Simmons-Duffin, N. Su, A. \\ Vichi \\ arXiv:1912.03324
}

\section{Recommended with a Commentary by Slava Rychkov, IHES (Bures-sur-Yvette)}

Although continuous phase transitions have been understood since many years, ${ }^{*}$ precise calculations of critical exponents using Wilson's renormalization group (RG) remain a challenge. One such case is the $\lambda$-point "anomaly" - a decade-old disagreement between Monte Carlo and experiment in the ${ }^{4} \mathrm{He}$ superfluid transition, which RG has been unable to settle. More recently, developments in the conformal bootstrap gave a new method to compute critical exponents and sparkled a renewed interest in the theory of phase transitions. Using the bootstrap, the featured paper (CLLPSSV) provided decisive evidence in the $\lambda$-point dispute. This gives us a good occasion to review the current state of the bootstrap approach.

Anomaly. The $\lambda$-point anomaly concerns the critical exponent $\nu$, whose experimental value $\nu^{\mathrm{EXP}}$ is incompatible with the Monte Carlo determination $\nu^{\mathrm{MC}}$ :

$$
\begin{aligned}
& \nu^{\mathrm{MC}}=0.67169(7)= \\
& \nu^{\mathrm{EXP}}=0.6709(1)=
\end{aligned}
$$

Figure 1: Determinations of $\nu$ in the $\mathrm{O}(2)$ model with their error bars.

The experimental value comes from very precise specific heat measurements by Lipa et al (2003) [1], on Earth and on board of space shuttle Columbia to remove pressure gradients causing gravitational rounding. Going to extremely small reduced temperatures $|t|=5 \times$ $10^{-10}$, they extracted $\alpha^{\mathrm{EXP}}=-0.0127(3)$. The quoted $\nu^{\mathrm{EXP}}$ then follows via the hyperscaling relation $\alpha=2-d \nu, d=3$.

*Foundations of the theory of phase transitions were laid down by Landau (1937), and the effects of fluctuations, important near critical points, were incorportated via the renormalization group (Wilson, 1971), allowing theoretical computations of critical exponents. In the 70's and 80's, many experimental measurements verified Wilson's theory. Nowadays, critical exponents are routinely computed via Monte Carlo simulations, with accuracy often superior to experiments and renormalization group calculations. 
On the other hand, Monte Carlo simulations of lattice models in the $\mathrm{O}(2)$ universality class of the $\lambda$-point transition systematically give much higher results, such as the quoted recent value $\nu^{\mathrm{MC}}$ from Hasenbusch (2019) [2]. The 8 standard deviations discrepancy of $\nu^{\mathrm{MC}}$ and $\nu^{\mathrm{EXP}}$ persists since $2006[3]$.

Is this an experimental or a theoretical error, or a hint of a new effect? Existing RG calculations are not precise enough to tell: $\nu^{\mathrm{RG}}$ has a larger error ${ }^{\dagger}$ and is comfortably consistent with both $\nu^{\mathrm{EXP}}$ and $\nu^{\mathrm{MC}}$ (Fig. 1).

Conformal bootstrap. The conformal bootstrap ${ }^{\ddagger}$ views the critical state as a superposition of infinitely many fluctuating fields $A_{i}(x)$, with powerlaw autocorrelation functions:

$$
\left\langle A_{i}(r) A_{i}(0)\right\rangle=\frac{1}{r^{2 \Delta_{i}}}, \quad \Delta_{i}=\text { scaling dimenson of } A_{i} .
$$

The cross-correlation 2-point functions vanish. ${ }^{\S}$ One works at asymptotically long distances, where the scale and conformal invariances are exact.

The fields $A_{i}$ and their scaling dimensions $\Delta_{i}$ can be identified with the eigenvectors and eigenvalues of renormalization group linearized around the fixed point. So each universality class has its own set of $\Delta_{i}$ 's. Dimensions $\Delta_{i}<d$ parametrize the relevant deformations of the fixed point; they are related to the most commonly encountered critical exponents via simple algebraic formulas. " Higher $\Delta_{i}$ 's correspond to increasingly more and more irrelevant deformations. It should be emphasized that these identifications between bootstrap and RG objects are only needed for the physical interpretation of bootstrap results. We will now review the two main principles underlying the actual bootstrap computations.

Operator Product Expansion (OPE). This says that the product of any two nearby fields can be expanded in the basis of fields $A_{i} . \|$ Schematically, we have:

$$
A_{i}(x) A_{j}(y)=\sum_{k=1}^{\infty} \frac{\lambda_{i j k}}{|x-y|^{\Delta_{i}+\Delta_{j}-\Delta_{k}}} A_{k}\left(\frac{x+y}{2}\right)+\ldots
$$

This is understood as an identity inside correlation functions. The power of $|x-y|$ is fixed by scaling, while the $\lambda_{i j k}$ are numerical coefficients which depend on the universality class. The ... terms in (2) involve first, second etc derivatives of the fields $A_{k}$. We don't write them because they are fixed by conformal invariance.

The OPE is powerful because it allows us to compute any correlator of the theory, recursively reducing $n$-point correlators to $(n-1),(n-2), \ldots, 2$-point correlators, the latter being known by Eq. (1). Of course this assumes that we know two infinite sets of numbers $\left\{\Delta_{i}\right\}$ and $\left\{\lambda_{i j k}\right\}$.

\footnotetext{
${ }^{\dagger}$ Guida and Zinn-Justin (1998) [4], Table 1, from Borel-resummed 6-loop calculations in the $\mathrm{O}(2)|\varphi|^{4}$ theory. There has been no significant progress in precision since 20 years.

${ }^{\ddagger}$ First mentioned in this journal club 5 years ago by late Leo Kadanoff [5]. For reviews see [6], [7].

$\S$ These are equal-time spatial correlation functions at thermal equilibrium in infinite flat space. Vector, tensor etc, fields are also taken into account, but here for simplicity we focus on scalars.

IE.g. $\quad \nu=1 /\left(d-\Delta_{s}\right)$ where $s$ is the temperature perturbation (the lowest singlet under the global symmetry of the critical point). For the $\mathrm{O}(2)$ model $s=|\varphi|^{2}$, the lowest charge 0 scalar field.

"More generally, any fluctuation localized in a finite region can be expanded into the basis of fields $A_{i}$. This basis property is only true if we include all fields on equal footing. E.g. both relevant and irrelevant fields. Also, both "fundamental fields" like $\varphi$, and "composite fields" like $\varphi^{2}$. In fact conformal bootstrap does not distinguish between fundamental and composite fields.
} 
Crossing. There are several ways to reduce a given $n$-point correlator to 2-point correlators, differing by the order of applying OPE. Crossing says that all ways should agree among themselves. For reasons having to do with conformal invariance, crossing is automatic for three fields, but it becomes non-trivial from four fields on. E.g., correlator of any four fields $\left\langle A_{1}\left(x_{1}\right) A_{2}\left(x_{2}\right) A_{3}\left(x_{3}\right) A_{4}\left(x_{4}\right)\right\rangle$ can be computed using the OPEs $A_{1} \times A_{2}$ and $A_{3} \times A_{4}$ or, alternatively, $A_{2} \times A_{3}$ and $A_{1} \times A_{4}$, and the two methods should agree. Schematically:



That the two expansions agree provides a nontrivial constraint, which can be expressed as a system of nonlinear equations for unknowns $\left\{\Delta_{i}\right\}$ and $\left\{\lambda_{i j k}\right\}$. Analyzing this system numerically, one excludes vast regions of parameter space where the solution does not exist (provably), and one is left with small regions where the scaling dimensions and OPE coefficients are therefore forced to belong.

Why should one believe the conformal bootstrap assumptions? The existence of the spectrum of scaling dimensions is one of the basic conclusions of Wilson's RG and is borrowed from there. Reality of scaling dimensions follows from the reflection positivity of the microscopic $\mathrm{O}(2)$ lattice model. Convergent OPE is a simple consequence of locality (the ability to cut and glue the system along surfaces, known in mathematics literature on constructive field theory as "Markoff property"), and scale invariance. Crossing is another consequence of locality. The system of bootstrap axioms thus appears reasonable and logically consistent, although at present it has not been derived mathematically from the basic postulates of statistical mechanics.

Conformal bootstrap for ${ }^{4} \mathrm{He}$. The $\lambda$-point transition is believed to be described by the fixed point of the $\mathrm{O}(2)$ model, and it has three relevant scalar fields, denoted by $\phi^{a}, s=\phi^{a} \phi_{a}$ and $t^{a b}=\phi^{a} \phi^{b}-\frac{1}{2} \delta^{a b}\left(\phi^{c} \phi_{c}\right)$ in the Landau-Ginzburg-Wilson approach where $a=1,2$ is the $\mathrm{O}(2)$ index. The field $\phi^{a}$ is the order parameter (the phase of the superfluid condensate wavefunction $\varphi=\phi^{1}+i \phi^{2}$ ), the field $s$ is the temperature perturbation, and the symmetric traceless $t^{a b}$ is a composite field describing local fluctuations of charge \pm 2 under the particle number conservation symmetry $U(1)$. Based on what is known about the $\mathrm{O}(2)$ model, CLLPSSV assume that all other scalars of charge 0,1,2,4 are irrelevant. They also assume that charge 3 scalars have dimension above 1 . These assumptions are conservative** and easy to impose in the bootstrap by restricting dimensions of operators appearing in the OPE. By these robust assumptions one selects the $\mathrm{O}(2)$ model from infinitely many other critical points possessing $U(1)$ invariance.

Under these assumptions, CLLPSSV study crossing equations for those 4-point correlators of the relevant fields $\phi, s, t$ which can be nonzero by charge conservation. There are seven such correlators: $\langle\phi \phi \phi \phi\rangle,\langle t t t t\rangle,\langle t t \phi \phi\rangle,\langle s s s s\rangle,\langle\phi \phi s s\rangle,\langle t t s s\rangle,\langle t s \phi \phi\rangle$. Crossing equations for different correlators involve the same quantities (e.g. $s$ appears in the OPE $\phi \times \phi$, while $\phi$ appears in the OPE $s \times \phi$, with the same OPE coefficient), and it's important to study them

\footnotetext{
${ }^{* *}$ E.g. $\varphi^{3}$ is known to have dimension close to 2.1 .
} 
concurrently. Simultaneous analysis of such a large number of correlators has not hitherto been attempted, and in fact CLLPSSV is the most complex conformal bootstrap study so far. It required numerous algorithmic improvements and took about $10^{6} \mathrm{CPU}$ hours on a supercomputer cluster (the developed software is publicly available).

CLLPSSV calculations give a precise determination of scaling dimensions of operators $\phi, s, t$, their OPE coefficients, some irrelevant operator dimensions, and a host of other quantities characterizing the $O(2)$ model (e.g. the zero-temperature conductivity $\sigma_{\infty}$ ). I will quote here only their answer for $\Delta_{s}$ and for $\nu=1 /\left(d-\Delta_{s}\right)$ :

$$
\Delta_{s}=1.51136(22) \quad \Longrightarrow \quad \nu^{\mathrm{CB}}=0.67175(10) .
$$

This nicely overlaps with $\nu^{\mathrm{MC}}$ and soundly excludes $\nu^{\mathrm{EXP}}$ (see Fig. 1).

Discussion. First a comment concerning the meaning of error bars. The error in $\nu^{\mathrm{RG}}$ comes from unknown higher order terms in the perturbative expansion and from arbitrary parameters in Borel resummation. The error in $\nu^{\mathrm{MC}}$ is a combination of a statistical Monte Carlo error and of a systematic error due to corrections to scaling. The error in $\nu^{\mathrm{EXP}}$ also has a statistical and a systematic component. The uncertainty in $\nu^{\mathrm{CB}}$ has a completely different origin. The total number of conformal bootstrap constraints is infinite. In any numerical bootstrap calculation we pick a finite subset of constraints, and find a region of parameters allowed by these constraints. The uncertainty is the size of the region allowed by the maximal set of constraints that one has been able to include so far (given computer resources). In the future, by including more constraints, one will be able to exclude larger and larger part of the parameter space, and the allowed region can therefore only shrink (it cannot jump to a non-overlapping position). This circumstance gives conformal bootstrap results the status of a final verdict.

Now that we know this, one still has to explain why $\nu^{\text {EXP }}$ is off. It might be a mundane experimental error affecting the experiment or the data analysis, ${ }^{\dagger \dagger}$ or it might be due to some physical effect. A theoretical error in the critical $\mathrm{O}(2)$ model no longer appears a viable option. ${ }^{\ddagger}$

More generally, the featured paper demonstrates the power of conformal bootstrap to give clearcut answers where renormalization group techniques are not sufficiently precise. As any practitioner knows, there are many other dark corners in the theory of critical phenomena where such clarification would be welcome. ${ }^{4} \mathrm{He}$ is just one example, and it is my expectation that many such verdicts will be pronounced in the coming years. It is also my dream that this theoretical activity may lead to a renewed interest in the experimental studies of critical phenomena.

\footnotetext{
${ }^{\dagger \dagger}$ However, the data is publicly available and there does not seem to be any problem in the fit nor in underestimating corrections to scaling (Junyu Liu and David Simmons-Duffin, personal communication).

$¥$ For a full list of options, we recall that the theory/experiment comparison needed the relation $\nu=$ $1 /\left(d-\Delta_{s}\right)$ and the hyperscaling relation $\alpha=2-d \nu$. Indeed, the bootstrap and Monte Carlo simulations extract the scaling dimension of the thermal perturbation $s$ working at criticality (bootstrap in infinite volume and Monte Carlo via finite-volume scaling), while the specific heat measurement is concerned, in a sense, with the singular part of the two-point function of $s$ slightly away from criticality. The expression for the correlation length exponent and the hyperscaling relation are some of the most basic consequences of the RG theory, and their failure in this case appears extremely unlikely.
} 


\section{References}

[1] J. A. Lipa, J. A. Nissen, D. A. Stricker, D. R. Swanson, and T. C. P. Chui, "Specific heat of liquid helium in zero gravity very near the lambda point," Phys. Rev. B 68 (Nov, 2003) 174518.

[2] M. Hasenbusch, "Monte Carlo study of an improved clock model in three dimensions," arXiv:1910.05916 [cond-mat.stat-mech].

[3] M. Campostrini, M. Hasenbusch, A. Pelissetto, and E. Vicari, "The Critical exponents of the superfluid transition in He-4," Phys. Rev. B74 (2006) 144506, arXiv: cond-mat/0605083.

[4] R. Guida and J. Zinn-Justin, "Critical exponents of the N vector model," J. Phys. A31 (1998) 8103-8121, arXiv: cond-mat/9803240 [cond-mat].

[5] L. P. Kadanoff, "Deep Understanding Achieved on the 3d Ising Model." https://www. condmatjclub. org/?p=2384.

[6] D. Poland and D. Simmons-Duffin, "The conformal bootstrap," Nature Phys. 12 no. 6, (2016) 535-539.

[7] D. Poland, S. Rychkov, and A. Vichi, "The Conformal Bootstrap: Theory, Numerical Techniques, and Applications," Rev. Mod. Phys. 91 (2019) 015002, arXiv:1805.04405 [hep-th]. 